\title{
Ell3 Modulates the Wound Healing Activity of Conditioned Medium of Adipose-derived Stem Cells
}

\author{
Jae-Yong Lee, Nuri Oh and ${ }^{\dagger}$ Kyung-Soon Park \\ Department of Biomedical Science, College of Life Science, CHA University, Seoul, Korea
}

\begin{abstract}
While adipose-derived stem cell-conditioned medium (ADSC-CM) has been demonstrated to promote skin wound healing, the mechanism regulating this effect remains unelucidated. In this study, we aimed to investigate the role of El13 in the wound healing activity of ADSC-CM. In vitro analysis revealed that Ell3 suppression in ADSCs impairs the promotive activity of ADSC-CM on the proliferation and migration of mouse embryonic fibroblasts (MEF) and normal human dermal fibroblasts (NHDF). Consistently, the expression of MMP family genes, which regulate cell proliferation and migration, was significantly suppressed in MEF and NHDF treated with siEll3-transfected ADSC-CM. Proinflammatory cytokines, such as interleukin-1 and interleukin-6, were highly expressed in MEF treated with siEll3-transfected ADSC-CM. The wound healing activity of siEll3-transfected ADSC-CM was significantly lower than that of the control in vivo. Our results suggest that Ell3 may contribute to the inhibition of inflammatory response during skin wound healing.
\end{abstract}

Key words : Adipose-derived stem cell-conditioned medium (ADSC-CM), El13, Wound healing, Prolinflammatory cytokines

\section{INTRODUCTION}

Because skin functions as a protective barrier against harmful environments, skin wounds must be repaired properly and efficiently. The wound healing process consists of complex, dynamic overlapping phases that include hemostasis, inflammation and tissue remodeling remodeling (Martin 1997; Eming et al., 2007; Guo \& dipietro, 2010) Wound healing progression depends on well-organized coordination between the wound site and the surrounding environment, such as the immune system and endogenous stem cells, including both dermal sheath stem cells and mesenchymal stem cells (Jahoda \& Reynolds, 2001; Gurtner et al., 2008; Wang et al., 2013). During the inflamma- tion phase, macrophages and neutrophils infiltrate the wound area and produce cytokines to promote the healing process. During the tissue formation and remodeling phase, fibroblast migration and proliferation and the expression of extracellular matrix components contribute to the process of skin repair.

While stem cells are reported to facilitate skin wound healing by enhancing neovascularization (Choi et al., 2013) and promoting microvascular remodeling (Dulmovits \& Herman, 2012), low cell survivability after transplantation to the wound site is a major limitation for the therapeutic application of stem cells (Modo et al., 2002). In addition, safety issues regarding the use of stem cells have not been completely resolved (Kucia et al., 2005). Because stem

\footnotetext{
Manuscript received September 22, 2017, Received in revised form September 25, 2017, Accepted September 26, 2017

${ }^{\dagger}$ Corresponding Author : Kyung-Soon Park, PhD, Department of Biomedical Science, College of Life Science and CHA Stem Cell Institute, CHA University, 335 Pangyo-ro, Bundang-gu, Seongnam 463-400, Korea. Tel: +82-31-881-7144, Fax: +82-31-881-7249, E-mail: kspark@cha.ac.kr

This is an Open Access article distributed under the terms of the Creative Commons Attribution Non-Commercial License (http:// creativecommons.org/licenses/by-nc/3.0) which permits unrestricted non-commercial use, distribution, and reproduction in any medium, provided the original work is properly cited.
} 
cell-conditioned medium (SC-CM) contains a multitude of trophic and survival signals, such as growth factors, chemokines and cytokines (Chen et al., 2009), using SC-CM in wound healing is an alternative approach to overcome the limitations of cell-based therapy. For example, paracrine factors secreted by adipose-derived stem cells (ADSCs) are reported to influence wound re-epithelization by promoting keratinocyte migration in vivo and by functioning as immunosuppressants (Chen et al., 2008; Park et al., 2008). Though, paracrines are known to be a beneficial effect of ADSCs, very little is known about the regulatory mechanism controlling the expression of ADSC paracrine factors.

Ell3 is a transcription elongation factor that belongs to the eleven-nineteen lysine-rich leukemia (ELL) family (Miller et al., 2000). In mouse embryonic stem cells, Ell3 is a super elongation complex component that mediates RNA polymerase II occupancy at enhancer of developmentrelated genes, which primes future gene activation following differentiation induction signals (Lin et al., 2013). In estrogen receptor-positive (ER+) breast cancer cells, Ell3 forms a transcriptional complex with ER, GATA3 and FOXA1 that regulates the expression of interleukin-20 (IL20 ), which leads to the stabilization of p53 and the chemosensitization of cancer cells upon treatment with cisdiamminedichloroplatinum (CDDP) (Ahn et al., 2015; Lee et al., 2017).

The molecular mechanism related to the tissue repair is still largely unknown. Recent investigations of wound healing mechanism revealed that wound healing process is involved with inflammatory response and there is a continual competition between pro-inflammatory and antiinflammatory signals during the process (Eming et al., 2007; Eming et al., 2010 ). Because Ell3 regulates the expression of IL-20 that is reportedly related to epithelial wound healing (Kolumam et al., 2017; Zhang et al., 2017), we questioned whether the expression level of Ell3 is associated with the wound healing activity of the secretome of ASC by regulating other cytokines including pro-inflam- matory cytokine, IL-1 and IL-6 (Slaats et al., 2016).

Therefore, we studied whether Ell3 regulates the efficiency of ADSC-CM to heal skin wounds in vitro and in vivo. We found that Ell3 suppression in ADSCs decreases the therapeutic efficacy of ADSC-CM with respect to dermal fibroblast migration and proliferation in vitro and wound healing activity in vivo. We report that Ell3 is related to the expression of VEGF and MMP family genes, which are prominent wound healing-related ADSC factors.

\section{MATERIALS AND METHODS}

\section{Cell culture and Ell3 knockdown}

ADSCs (SEFO, Seoul, Korea) were grown in alpha-Minimum Essential Media (a-MEM, Gibco, MA, USA) supplemented with $10 \%$ fetal bovine serum (FBS) (Gibco) and $1 \%$ penicillin/streptomycin (Invitrogen, CA, USA). Mouse embryonic fibroblasts (MEF) and normal human dermal fibroblasts (NHDF) were grown in Dulbecco's Modified Eagle's Medium (DMEM, Gibco) supplemented with FBS and $1 \%$ penicillin/streptomycin. All cell lines were incubated at $37^{\circ} \mathrm{C}$ and $5 \% \mathrm{CO}_{2}$. Non-specific control siRNA (siNS, D-001810-10) and human Ell3 siRNA (siEll3, L014601-02-0010) were purchased from Dharmacon, Inc. (IL, USA). For Ell3 knockdown, ADSCs were transfected with $100 \mathrm{nM}$ siNS or $100 \mathrm{nM}$ siEll3 using lipofectamine 3000 (Invitrogen,CA, USA), and the cells were harvested 48 hours after transfection for subsequent analyses.

\section{RNA extraction and real-time RT-PCR}

Total RNA was extracted using TRIzol (Invitrogen,CA, USA), and $5 \mu \mathrm{g}$ of total RNA was used to synthesize cDNA according to the manufacturer's instructions. Quantitative gene expression analysis was performed using real-time RT-PCR with the primer pairs listed in Table 1.

\section{Preparation of ADSC-CM}

Briefly, 24 hours after siRNA transfection, normal ADSC 
Table 1. Primers used in this study

\begin{tabular}{|c|c|c|}
\hline Primer & & Human sequence (5' to 3') \\
\hline \multirow{2}{*}{ GAPDH } & $\mathrm{F}$ & ACC CAG AAG ACT GTG GAT GG \\
\hline & $\mathrm{R}$ & TCT AGA CGG CAG GTC AGG TC \\
\hline \multirow{2}{*}{ ELL3 } & $\mathrm{F}$ & CTA CAA GGC CTG ACC AAT CAG G \\
\hline & $\mathrm{R}$ & CTG GAG TTC CTC GCC GAA c \\
\hline \multirow{2}{*}{ MMP1 } & $\bar{F}$ & AGCTAGCTCAGGATGACATTGATG \\
\hline & $\mathrm{R}$ & GCCGATGGGCTGGACAG \\
\hline \multirow{2}{*}{ MMP2 } & $\mathrm{F}$ & TCT CCT GAC ATT GAC CTT GGC \\
\hline & $\mathrm{R}$ & CAA GGT GCT GGC TGA GTA GAT C \\
\hline \multirow{2}{*}{ MMP3 } & $\mathrm{F}$ & ATT CCA TGG AGC CAG GCT TTC \\
\hline & $\mathrm{R}$ & CAT TTG GGT CAA ACT CCA ACT GTG \\
\hline \multirow{2}{*}{ MMP9 } & $\mathrm{F}$ & TTG ACA GCG ACA AGA AGT GG \\
\hline & $\mathrm{R}$ & GCC ATT CAC GTC GTC CTT AT \\
\hline \multirow{2}{*}{ MMP10 } & $\mathrm{F}$ & ATTTTGGCCCTCTCTTCCA \\
\hline & $\mathrm{R}$ & TTGTCCGCTGCAAAGAAGTA \\
\hline \multirow{2}{*}{ MMP13 } & $\mathrm{F}$ & TCC CAG GAA TTG GTG ATA AAG TAG A \\
\hline & $\mathrm{R}$ & CTG GCA TGA CGC GAA CAA TA \\
\hline \multirow{2}{*}{ VEGF } & $\mathrm{F}$ & GAG ATG AGC TTC CTA CAG CAC \\
\hline & $\mathrm{R}$ & TCA CCG CCT CGG CTT GTC ACA T \\
\hline \multirow{2}{*}{ IL-1 } & $\mathrm{F}$ & AAA CAG ATG AAG TGC TCC TTC CAG G \\
\hline & $\mathrm{R}$ & TGG AGA ACA CCA CTT GTT GCT CCA \\
\hline \multirow{2}{*}{ IL-6 } & $\mathrm{F}$ & CCA GCT ATG AAC TCC TTC TC \\
\hline & $\mathrm{R}$ & GCT TGT TCC TCA CAT CTC TC \\
\hline $\mathrm{COL}$ & $\mathrm{F}$ & CGG TGT GAC TCG TGC AGC \\
\hline $1 \mathrm{~A} 1$ & $\mathrm{R}$ & ACA GCC GCT TCA CCT ACA GC \\
\hline $\mathrm{CO}$ & $\mathrm{F}$ & TCA AAC TGG CTG CCA GCA T \\
\hline $\mathrm{L} 1 \mathrm{~A} 2$ & $\mathrm{R}$ & CAA GAA ACA CGT CTG GCT AGG \\
\hline $\mathrm{COL}$ & $\mathrm{F}$ & CGG CTT CCA CAC ATC CTT AT \\
\hline $2 \mathrm{~A} 1$ & $\mathrm{R}$ & CTG TCC TTC GGT GTC AGG G \\
\hline \multirow{2}{*}{ GAPDH } & $\mathrm{F}$ & TCA ACA GCA ACT CCC ACT CTT CCA \\
\hline & $\mathrm{R}$ & TTG TCA TTG AGA GCA ATG CCA GCC \\
\hline \multirow{2}{*}{ MMP1 } & $\mathrm{F}$ & ACT ACA TTT AGG GGA GAG GTG T \\
\hline & $\mathrm{R}$ & GCA GCG TCA AGT TTA ACT GGA A \\
\hline \multirow{2}{*}{ MMP2 } & $\mathrm{F}$ & CAA GTT CCC CGG CGA TGT C \\
\hline & $\mathrm{R}$ & TTC TGG TCA AGG TCA CCT GTC \\
\hline \multirow{2}{*}{ MMP3 } & $\mathrm{F}$ & ACA TGG AGA CTT TGT CCC TTT TG \\
\hline & $\mathrm{R}$ & TTG GCT GAG TGG TAG AGT CCC \\
\hline \multirow{2}{*}{ MMP9 } & $\mathrm{F}$ & CTG GAC AGC CAG ACA CTA AAG \\
\hline & $\mathrm{R}$ & CTC GCG GCA AGT CTT CAG AG \\
\hline \multirow{2}{*}{ MMP10 } & $\mathrm{F}$ & ACC CAA GGA CTC CAG CAA TG \\
\hline & $\mathrm{R}$ & TGG AAA GAG ATT GCA AAG TGA ATG \\
\hline \multirow{2}{*}{ MMP13 } & $\mathrm{F}$ & AAG ATG TGG AGT GCC TGA TG \\
\hline & $\mathrm{R}$ & AAG GCC TTC TCC ACT TCA GA \\
\hline \multirow{2}{*}{ VEGF } & $\mathrm{F}$ & AAC GAT GAA GCC CTG GAG TG \\
\hline & $\mathrm{R}$ & TGA GAG GTC TGG TTC CCG A \\
\hline
\end{tabular}

Table 1. Continued

\begin{tabular}{ccc}
\hline \hline Primer & & Mouse sequence (5' to 3') \\
\hline \multirow{2}{*}{ COL1A1 } & F & ACT GCA ACA TGG AGA CAG GTC AGA \\
& R & ATC GGT CAT GCT CTC TCC AAA CCA \\
\hline \multirow{2}{*}{ COL1A2 } & F & GGA GGG AAC GGT CCA CGA T \\
& R & GAG TCC GCG TAT CCA CAA \\
\hline \multirow{2}{*}{ COL2A1 } & F & TTC CTC CGT CTA CTG TCC ACT GA \\
& R & CTA CAT CAT TGG AGC CCT GGA T \\
\hline
\end{tabular}

culture media was replaced with serum-free media (SFM a-MEM), and the cells were incubated for an additional 48 hours. The culture media was harvested and centrifuged at 1,000 rpm for 3 minutes, and the supernatant was collected and filtrated with a $0.45-\mu \mathrm{m}$ syringe filter for the experiment.

\section{Cell proliferation assays}

Briefly, $4 \times 10^{4}$ MEF or NHDF were cultured in CM prepared from siNS- or siEll3-transfected ADSCs, and cell numbers were counted every 24 hours for 4 days using a hemocytometer to analyze the effects of $\mathrm{CM}$ on cell proliferation. All samples were assayed in triplicate, and the means for each experiment were calculated.

\section{Scratch/wound healing assay and transwell mi-} gration assay

The effects of ADSC-CM on the migration potential of MEF and NHDF was assessed using the scratch/wound healing and transwell migration assays. For the scratch/wound healing assay, $1 \times 10^{5}$ MEF or NHDF were seeded onto 6well plates, and mitomycin C (10 $\mu \mathrm{g} / \mathrm{mL})$ (Sigma Aldrich, MI, USA) was added for 2 hours when the cell densities reached $\sim 90 \%$ to prevent cell proliferation. After the cells were washed with SFM, the monolayers were wounded using a micropipette tip. The cells were cultured in CM for 2 days, and the degree of cell wounding was measured every 24 hours using a microscope. For the transwell migration assay, $2 \times 10^{3}$ MEF or NHDF were seed onto the upper well of a transwell plate $(6.5 \mathrm{~mm}$ diameter, $8 \mu \mathrm{m}$ pores; Corning, NY, USA). The lower portion of the well 
contained CM derived from siNS- or siEll3-transfected ADSCs. After incubation for $24 \mathrm{~h}$, the cells were fixed in 4\% paraformaldehyde (Santa Cruz, CA, USA) and stained with crystal violet (Sigma Aldrich).

\section{In vivo wound healing model}

Balb/c mice (8-week-old males, body weight: 20-23 g) were obtained from Orient, Inc. (Seong-Nam, Korea). After hair removal, the skin of the mice was wounded using a punch, and the wounded spots were treated with $100 \mu \mathrm{L}$ of CM prepared from siNS- or siEll3-transfected ADSCs; SFM was used as the negative control. Wounded mouse skin tissues were harvested 7 days after CM treatment and then fixed in $4 \%$ formaldehyde.

\section{H\&E staining}

Skin tissues fixed in $4 \%$ buffered paraformaldehyde were paraffinized and sectioned into $5-\mu \mathrm{m}$-thick sections. The sections were stained with hematoxylin and eosin (H\&E) and examined under a Nikon COOLSCOPE (Nikon Instruments, Inc., NY, USA).

\section{Statistical analysis}

The data are presented as means $\pm \mathrm{SE}$. Data were analyzed using Student's $t$-test. Differences were considered statistically significant at $P<0.05$.

\section{RESULTS AND DISCUSSION}

To study the role of Ell3 in the secretome activity of ADSCs to repair skin wounds, we first suppressed Ell3 expression using a siRNA targeting Ell3 in ADSCs. As shown in Fig. 1A, Ell3 expression was suppressed up to $50 \%$ by siRNA transfection. We used media harvested from ADSCs transfected with siNS or siEll3 for 24 hours (siNS CM or siEll3 $\mathrm{CM}$ ) as MEF culture media to examine the effects of $\mathrm{CM}$ on the proliferation of mouse fibroblasts. The proliferation rate of MEF cultured in siEll3 CM was significantly decreased compared to that of MEF cultured in siNS CM (Fig. 1B). The migration rate of MEF cultured in siEll3 CM was lower than that of MEF cultured in siNS $\mathrm{CM}$, as determined by the scratch wound healing assay (Fig. 1C). The transwell assay also revealed that the migration ability of MEF cultured in siEll3 CM was lower than that of MEF cultured in siNS CM (Fig. 1D). Consistently, the expression of MMP family genes, which regulate diverse cell functions, including proliferation and migration, was lower in ADSCs cultured in siEll3 CM than in those cultured in siNS CM (Fig. 1E).

We next examined whether the results observed in mouse fibroblasts were reproduced in human fibroblasts. Similar to MEF, the proliferation rate of NHDF cultured in siEll3 CM was lower than that of NHDF cultured in siNS CM (Fig. 2A). The scratch/wound healing and transwell assays confirmed that Ell3 knockdown could suppress the ability of ADSC-CM to promote NHDF migration (Fig. 2B,C). Consistent with the results in MEF, the expression of MMP family genes in NHDF was significantly lower when cells were cultured in siEll3 CM (Fig. 2D).

Based on these results, we concluded that Ell3 suppression in ADSCs diminishes the ability of CM to promote fibroblast proliferation and migration, which are two prominent in vitro parameters to estimate wound healing ability.

To evaluate the effect of Ell3 suppression on the wound healing ability of ADSC-CM in vivo, we applied siNS CM or siEll3 CM to mouse skin wounds that were induced by punch (Fig. 3A). SFM was applied to the wounds as a negative control, and the degree of open, fully thick excisional wound contraction was observed. As shown in representative lesions that were assessed 7 days after CM or SFM application, skin wounds treated with siEll3 CM recovered to a lesser extent than those treated with SFM (Fig. 3B). Assessing wound repair efficiency by examining the relative wound area on the indicated days after the application of CM or SFM showed that siEll3 CM could not enhance the wound healing rate, whereas siNS CM significantly 
A.

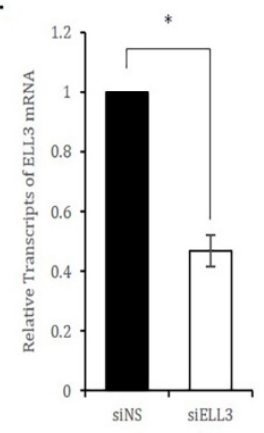

B.

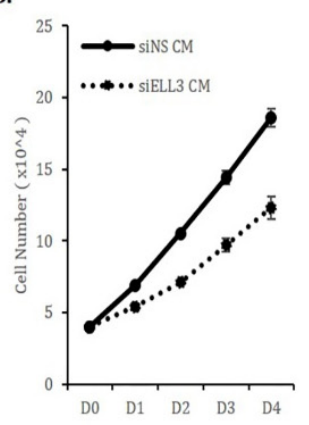

C.

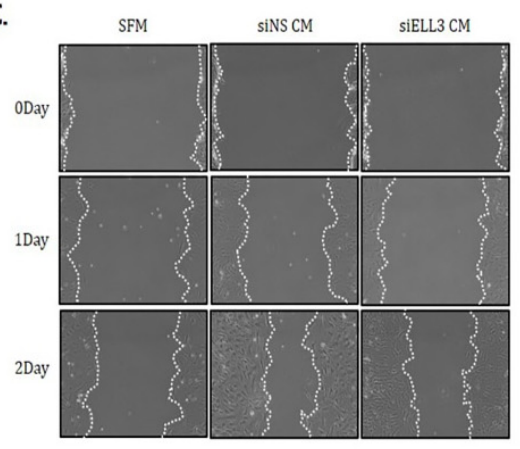

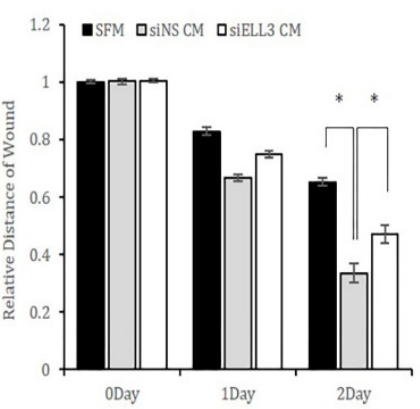

D.

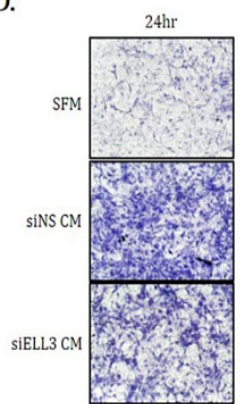

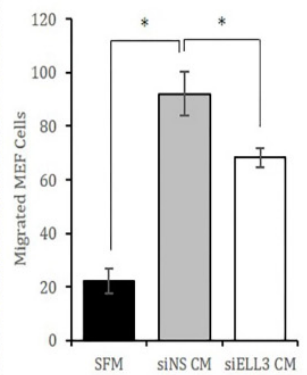

E.

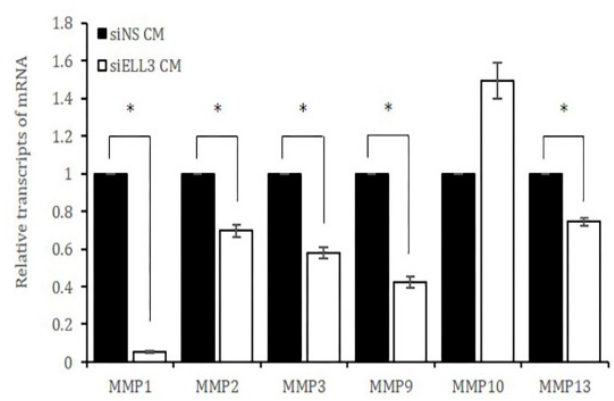

Fig. 1. Effects of Ell3-suppressed ADSC-CM on the proliferation, migration and migration-related marker expression of mouse embryonic fibroblasts (MEF) in vitro. (A) siRNA-mediated suppression of Ell3 in ADSCs. Ell3 expression was quantitatively analyzed by real-time RT-PCR 48 hours after siRNA transfection. (B) The proliferation of MEF cultured in CM collected from siNS- or siEll3-transfected ADSCs was analyzed by counting cells on the indicated days after seeding. (C) The migration ability of MEF cultured in CM collected from siNS- or siEll3transfected ADSCs was analyzed by the scratch/wound assay. Cell migration was photographed on the indicated days after scratching (left), and the relative migration ability was quantified by subtracting the cell-free areas on days 1 and 2 from the cell-free area on day 0 (right). (D) The migration ability of MEF was analyzed by the transwell assay as described in the Materials and Methods. Images were captured at 24 hours under 40× magnification (left). Cells were counted from 10 randomly selected fields, and the averages were calculated (right). (E) Relative expression of MMP family genes in MEF treated with CM collected from ADSCs transfected with siNS or siEll3 for 24 hrs. Abbreviations: siNS, non-specific siRNA; siEll3, siRNA targeting Ell3; CM, conditioned media; SFM, serum-free media; MEF, mouse embryonic stem cells. The error bars represent the standard errors from three independent experiments, which were each performed using triplicate samples. ${ }^{*} P<0.05$ (Student's $t$-test).

promoted wound repair (Fig. 3C). In addition, H\&E staining of wounded skin tissue revealed that siEll3 CM promoted inflammation (Fig. 3C). Because these results support the possibility that siEll3 CM promotes the expression of proinflammatory cytokines in fibroblasts, we analyzed the effect of Ell3 suppression on the expression of proinflammatory cytokines, including interleukin-1 (IL-1) and interleukin-6 (IL-6), in MEF treated with siNS CM or siEl13 CM. As expected, the expression levels of IL-1 and IL-6 were significantly increased in MEF treated with siEll3 CM compared to the levels in those treated with the control (Fig. 3D). Overall, our work further elucidates the role of Ell3 in the wound healing efficiency of ADSC-CM, although the downstream target genes regulated by Ell3 re- 
A.

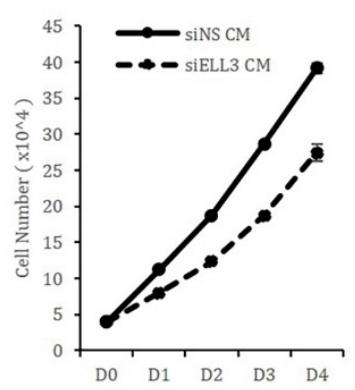

C.

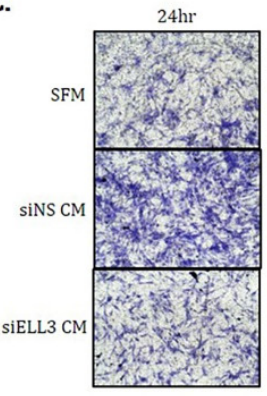

B.

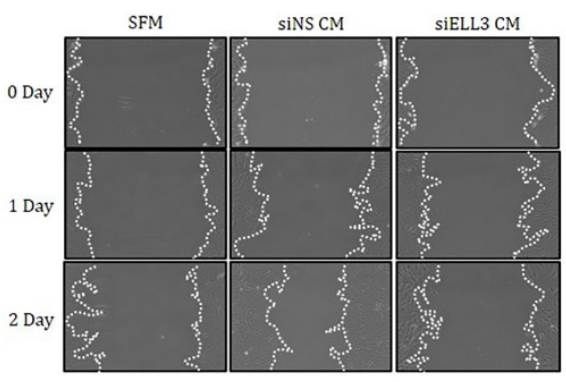

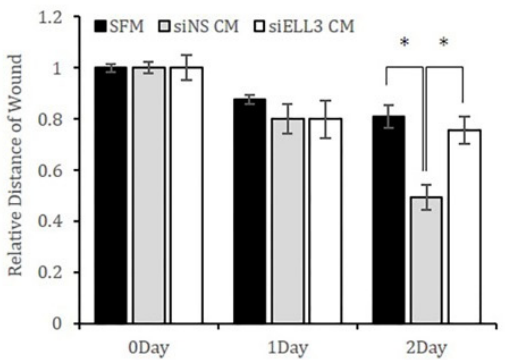

D.
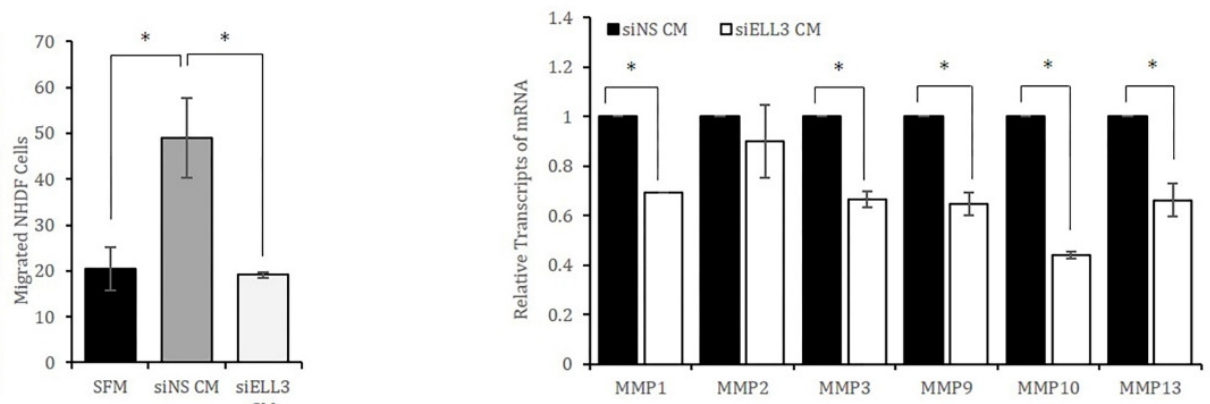

Fig. 2. Effects of Ell3-suppressed ADSC-CM on the proliferation, migration and migration-related marker expression of normal human dermal fibroblasts (NHDF) in vitro. (A) The proliferation of NHDF cultured in CM collected from siNS- or siEll3-transfected ADSCs was analyzed by counting cells on the indicated days after seeding. (B) The migration ability of NHDF cultured in CM collected from siNS- or siEll3-transfected ADSCs was analyzed by the scratch/wound assay. Cell migration was photographed on the indicated days after scratching (left), and the relative migration ability was quantified by subtracting the cell-free areas on days 1 and 2 from the cell-free area on day 0 (right). ${ }^{*} p<0.01$. (C) The migration ability of NHDF was analyzed by the transwell assay as described in the Materials and Methods. Images were captured at 24 hours under $40 \times$ magnification (left). Cells were counted from 10 randomly selected fields, and the averages were calculated (right). ${ }^{*} p<0.01$. (D) Relative expression of MMP family genes, a typical marker of migration, in NHDF treated with CM collected from ADSCs transfected with siNS or siEll3 for 24 hrs. Abbreviations: siNS, non-specific siRNA; siEll3, siRNA targeting Ell3; CM, conditioned media; SFM, serum-free media; MMP, matrix metalloproteinase. The error bars represent the standard errors from three independent experiments, which were each performed using triplicate samples. ${ }^{*} p<0.05$ (Student's $t$-test).

main to be identified.

\section{REFERENCES}

\section{ACKNOWLEDGEMENTS}

This work was supported by the Priority Research Centers Program through the National Research Foundation of Korea (NRF) (20120006679) and the Ministry of Education, Science, and Technology (NRF-2017-M3A9B4031169).
Martin P (1997) Wound healing--aiming for perfect skin regeneration. Science 276:75-81.

Eming SA, Krieg T, Davidson JM (2007) Inflammation in wound repair: molecular and cellular mechanisms. J Invest Dermatol 127:514-525.

Guo S, Dipietro LA (2010) Factors affecting wound heal 
A.

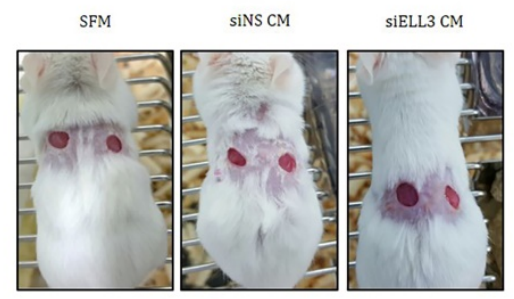

B.

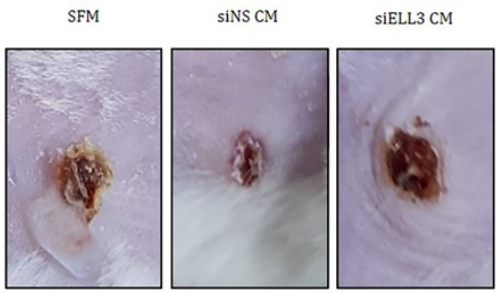

D.
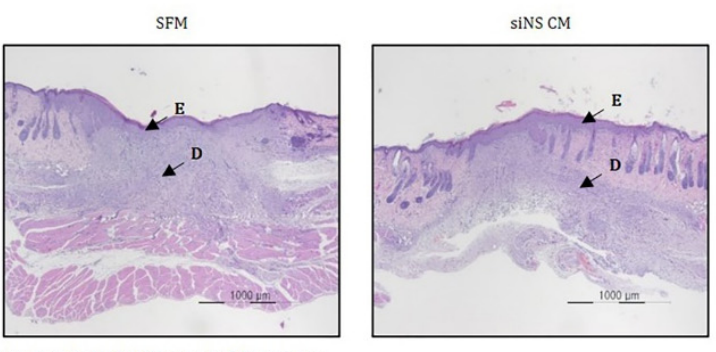

D : Dermis, E : Epi-Dermis, I : Inflammation
C.

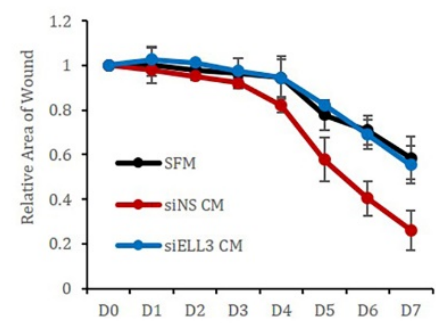

E.

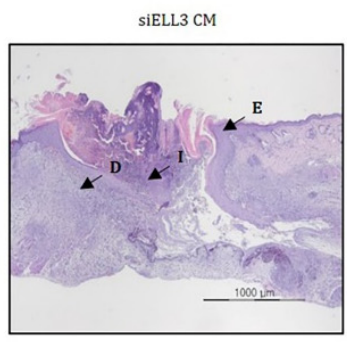

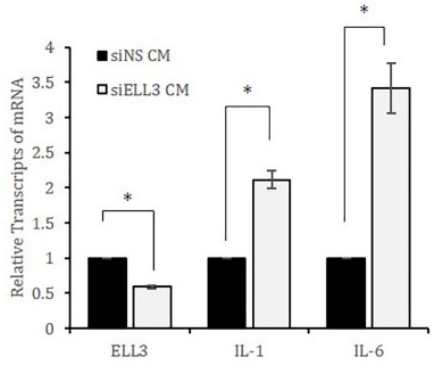

Fig. 3. Effects of Ell3 suppression on the in vivo wound healing activity of ADSC-CM. CM collected from siNS- or siEll3-transfected ADSCs was applied to mouse wounds every 24 hours for 7 days. Serum-free media (SFM) was applied as a negative control. (A) The backs of mice were wounded by punches. (B) Wound areas to which SFM and CM collected from siNS- and siEll3-transfected ADSCs were applied 7 days after treatment. (C) The wound area was quantitatively analyzed on the indicated days after treatment. (D) Sections of paraffin-blocked wounded tissues were stained with hematoxylin and eosin (H\&E). The arrows indicate E (epi-dermis), D (dermis), and I (inflammation). (E) Relative expression of IL-1 and IL-6 transcripts in ADSCs transfected with siNS- or siEll3- for 24 hrs.

ing. J Dent Res 89:219-229.

Gurtner GC, Werner S, Barrandon Y, Longaker MT (2008)

Wound repair and regeneration. Nature 453:314-321

Jahoda CA, Reynolds AJ (2001) Hair follicle dermal sheath cells: unsung participants in wound healing. Lancet 358:1445-1448.

Wang Y, Sun Y, Yang XY, Ji SZ, Han S, Xia ZF (2013)

Mobilised bone marrow-derived cells accelerate woundhealing. Int Wound J 10:473-9.

Choi M, Lee HS, Naidansaren P, Kim HK, O E, Cha JH, Ahn HY, Yang PI, Shin JC, Joe YA (2013) Proangiogenic features of Wharton's jelly-derived mesenchymal stromal/stem cells and their ability to form functional vessels. Int J Biochem Cell Biol 45:560-570.
Dulmovits BM, Herman IM (2012) Microvascular remodeling and wound healing: a role for pericytes. Int $\mathrm{J}$ Biochem Cell Biol 44:1800-1812.

Modo M, Rezaie P, Heuschling P, Patel S, Male DK, Hodges H (2002) Transplantation of neural stem cells in a rat model of stroke: assessment of short-term graft survival and acute host immunological response. Brain res 958: 70-82.

Kucia M, Reca R, Miekus K, Wanzeck J, Wojakowski W, Janowska-Wieczorek A, Ratajczak J, Ratajczak MZ (2005) Trafficking of normal stem cells and metastasis of cancer stem cells involve similar mechanisms: pivotal role of the SDF-1-CXCR4 axis. Stem cells 23:879-894.

Chen M, Przyborowski M, Berthiaume F (2009) Stem cells 
for skin tissue engineering and wound healing. Crit Rev Biomed Eng 37:399-421.

Chen L, Tredget EE, Wu PY, Wu Y (2008) Paracrine factors of mesenchymal stem cells recruit macrophages and endothelial lineage cells and enhance wound healing. PloS one 3: e1886.

Park BS, Jang KA, Sung JH, Park JS, Kwon YH, Kim KJ, Kim WS (2008) Adipose-derived stem cells and their secretory factors as a promising therapy for skin aging. Dermatol Surg 34:1323-1326.

Miller T, Williams K, Johnstone RW, Shilatifard A (2000) Identification, cloning, expression, and biochemical characterization of the testis-specific RNA polymerase II elongation factor ELL3. J Biol Chem, 275:3205232056.

Lin C, Garruss AS, Luo Z, Guo F, Shilatifard A (2013) The RNA Pol II elongation factor Ell3 marks enhancers in ES cells and primes future gene activation. Cell 152: 144-156.

Ahn HJ, Kim KS, Shin KW, Lim KH, Kim JO, Lee JY, Kim J, Park JH, Yang KM, Baek KH, Ko JJ, Park KS (2015) Ell3 stabilizes p53 following CDDP treatment via its effects on ubiquitin-dependent and -independent proteasomal degradation pathways in breast cancer cells. Oncotarget 6:44523-44537.
Lee JY, Park YJ, Oh N, Kwack KB, Park KS (2017) A transcriptional complex composed of ER(alpha), GATA3, FOXA1 and ELL3 regulates IL-20 expression in breast cancer cells. Oncotarget 8:42752-42760.

Eming SA, Koch M, Krieger A, Brachvogel B, Kreft S, Bruckner-Tuderman L, Krieg T, Shannon JD, Fox JW (2010) Differential proteomic analysis distinguishes tissue repair biomarker signatures in wound exudates obtained from normal healing and chronic wounds. J Proteome Res, 2010. 9:4758-4766.

Zhang W, Magadi S, Li Z, Smith CW, Burns AR (2017) IL-20 promotes epithelial healing of the injured mouse cornea. Exp Eye Res 154:22-29.

Kolumam G, Wu X, Lee WP, Hackney JA, Zavala-Solorio J, Gandham V, Danilenko DM, Arora P, Wang X, Ouyang W (2017) IL-22R Ligands IL-20, IL-22, and IL-24 Promote Wound Healing in Diabetic db/db Mice. PloS one 12:e0170639.

Slaats J, Ten Oever J, van de Veerdonk FL, Netea MG (2016) IL-1beta/IL-6/CRP and IL-18/ferritin: Distinct Inflammatory Programs in Infections. PLoS pathogens 12: e1005973.

Zhang W, Magadi S, Li Z, Smith CW, Burns AR (2017) IL-20 promotes epithelial healing of the injured mouse cornea. Exp Eye Res 154:22-29. 